\title{
Self-fulfillment Development among the Arab Sector in Israel
}

\begin{abstract}
Gith Emad
Correspondence: Gith Emad, Lecturer at The Sakhnin Academic College for Teacher Education, Senior Educational Psychologist and Director of the Psychological Services in Arraba Village, Israel; Department of Psychology, Law, and Ethics, International Center for Health, Law and Ethics, University of Haifa,Haifa, Israel.
\end{abstract}

Received: March 10, 2017

Accepted: April 5, 2017

Online Published: April 6, 2017

doi:10.11114/jets.v5i5.2339

URL: https://doi.org/10.11114/jets.v5i5.2339

\begin{abstract}
Self-determination and fulfillment are central components of personal development in both developing and developed societies. These terms are used frequently by many researchers, especially in the work by Maslow, who particularly emphasized this concept.

This article presents a roadmap of sorts that describes the factors that can leverage and promote a process that leads to self-fulfillment and the sources from where these factors are derived among Arab sector in Israel. Most importantly, it defines a professional protocol and its components. This is a route that will be certain to prove useful, understandable, and applicable for parents and professionals.
\end{abstract}

Keywords: Maslow scale, self-fulfillment scale, self-determination, self-image, self-esteem, self-awareness, fear, anxiety, impulsive behavior, social contacts, dependent behavior, organization, planning

\section{Introduction}

Educational psychologists have spent years attempting to understand how people can achieve a sense of self-fulfillment. The various models proposed emphasize belonging, meeting basic needs, and more.

Previous self-determination theory argued that the subject's needs are innate; these needs are defined as nutriments that are essential for people's survival, growth, and integrity (Ryan, Sheldon, Kasser, \& Deci, 1996). Several studies have mentioned that competence, autonomy, and relatedness are in fact essential needs. In a study by Ilardi, Leone, Kasser, and Ryan (1993), participants who experienced greater satisfaction of their needs for competence, autonomy, and relatedness reported more positive job attitudes, higher self-esteem, compared to participants who experienced low need satisfaction. In summary, it can be shown that the need for self-fulfillment is important for motivation and general well-being.

Maslow (1971) posited a hierarchy of human needs based on deficiency and growth needs. Within the deficiency needs, each lower need must be met before moving to the next higher level. Once each of these needs has been satisfied, if at some future time a deficiency is detected, the individual will act to remove the deficiency. The first four levels are physiological (hunger, thirst, bodily comforts, etc.), safety/security (out of danger), belongingness and love (affiliate with others and be accepted) and esteem (to achieve, be competent, and gain approval and recognition).

Maslow later differentiated the growth need of self-actualization, specifically identifying two of the first growth needs as part of the more general level of self-actualization (Maslow \& Lowery, 1998). He also identified another one beyond the general level that focused on growth beyond that oriented towards self (Maslow, 1971). They are cognitive (to know, to understand, and explore), aesthetic (symmetry, order, and beauty), self-actualization: (to find self-fulfillment and realize one's potential), and self-transcendence (to connect to something beyond the ego or to help others find self-fulfillment and realize their potential).

Mathes (1981) identified three levels of human needs: first, material (physiological and safety), second, social (belongingness and esteem), and third, spiritual. Mathes considered security and self-esteem as unwarranted. Alderfer (1972) developed a hierarchy with his ERG (existence, relatedness, and growth) theory. His approach modified Maslow's theory and incorporated concepts from systems theory into his work on personality.

Ryan \& Deci (2000) indicated three needs: the need for autonomy, the need for competence, and the need for relatedness. Thompson, Grace and Cohen (2001) postulated that the most important needs are connection, recognition, and power. Nohria, Lawrence, and Wilson (2001) indicated four basic needs: first, to acquire objects and experiences, second, to 
bond with others in long-term relationships, third, to learn and fourth, to defend ourselves. Pink (2009) suggested three basic needs: autonomy, mastery, and purpose.

As Self-determination theory has expanded, both theoretical developments and empirical findings have led researchers to examine a related to personality growth, effective functioning, and wellness.

Relatedness, safety, are a components of every theory, however, there are many factors which affect self-determination especially in modern time, for example: vocabulary and linguistic ability was found as related variable to self-awareness (Goodman ,2001). Similarly, coping successfully with fear and anxiety seems to be related to self-awareness Lewis and Ramsay (1997) indicated a relation between coping with anxiety or fear and self-recognition. In addition, Social interactions were found related to self-awareness and self-esteem (Steele 2010). Management and organization skills have postulated as important to self-awareness (Goleman, 1996). Impulsive behavior was related to self-awareness in previous research (De young, 2013). Absence of dependent behavior is related to lack of self-confidence and difficulty in making decisions (Goleman, 1996). Normal sexual conduct was found as related variable to self-recognition (Tolman, 2012).

Still, there is no agreement about the order and identification of basic human needs. Ryan and Deci (2000) suggested three needs, which are not necessarily arranged hierarchically: autonomy, competence, and relatedness. Other researchers have criticized Maslow's concept of basic human needs, including Thompson, Grace, and Cohen (2001), who suggested that the most essential needs are connection, recognition, and power.

For over two decades, like many other researchers, I used tools that were designed to teach parents how to contribute to the emotional and personal development of their children. Over the years, as I expanded my psychological expertise in various European countries and developed my professional skill as an Arab Israeli psychologist, I learned that the basic concepts used to define self-fulfillment are often interpreted differently among different populations and societies. As the director of an educational psychology service center that provides professional therapy and consultation services for a society that is classified as a collective one (Auther, 2014), namely the Arab society in Israel, I developed a new model. This approach has gradually evolved into a model that paves a new road and offers a narrative and sufficient tools that I believe can serve as an educational and psychological platform from which individuals, and specifically children, can successfully achieve a higher degree of self-fulfillment.

Nevertheless, the existing theories have been employed by the entire professional world of educational psychology for years and have been applied routinely to seek understanding of the behavioral world of children and their psychological experiences, as well as their emotional and personal needs.

This paper will present a model that carefully outlines a path that leads to self-fulfillment. The current study shows an approach that applies to Western and Eastern cultures alike and to any person that is exposed to family settings and experiences of any kind. It is important to note that the model presented here describes the entire journey, from beginning to end. Parents can make the role that they play more significant by using this model to develop communication with their children from a very young age, that is, from the onset of linguistic, intellectual behavioral and psychological development. This stage can be roughly defined as beginning when children are two to three years old and ending after adolescence. Parents play a central role in their children's development and have the ability to promote their children's ability to become sharing, aware, and communicative people. When slight delays or distortions in these areas occur, the child may grow up within an "unprotected family setting" (Author, 2013). It goes without saying that the various educational community settings impact a child's development through interaction that is based an understanding of the individual's basic, psychological, emotional, social, and behavioral needs (Author, 2014). Interaction that is flawed and insensitive to a child's needs is certain to impair the child's development at a very early stage.

Different developed societies will naturally define the parental role in different ways. At times, parental competence does not suffice and must be supported by other concepts of parenting. The first is parental readiness, and the second is parental responsibility (Author, 2013). As a therapist, I have seen that parents that are sufficiently prepared and responsible will be more competent. My experience has shown that the motivation to perform better as parents and to meet their children's needs does not always suffice (Author, 2013). This paper will describe a tool and a model that will develop a motivated parents' toolkit. 


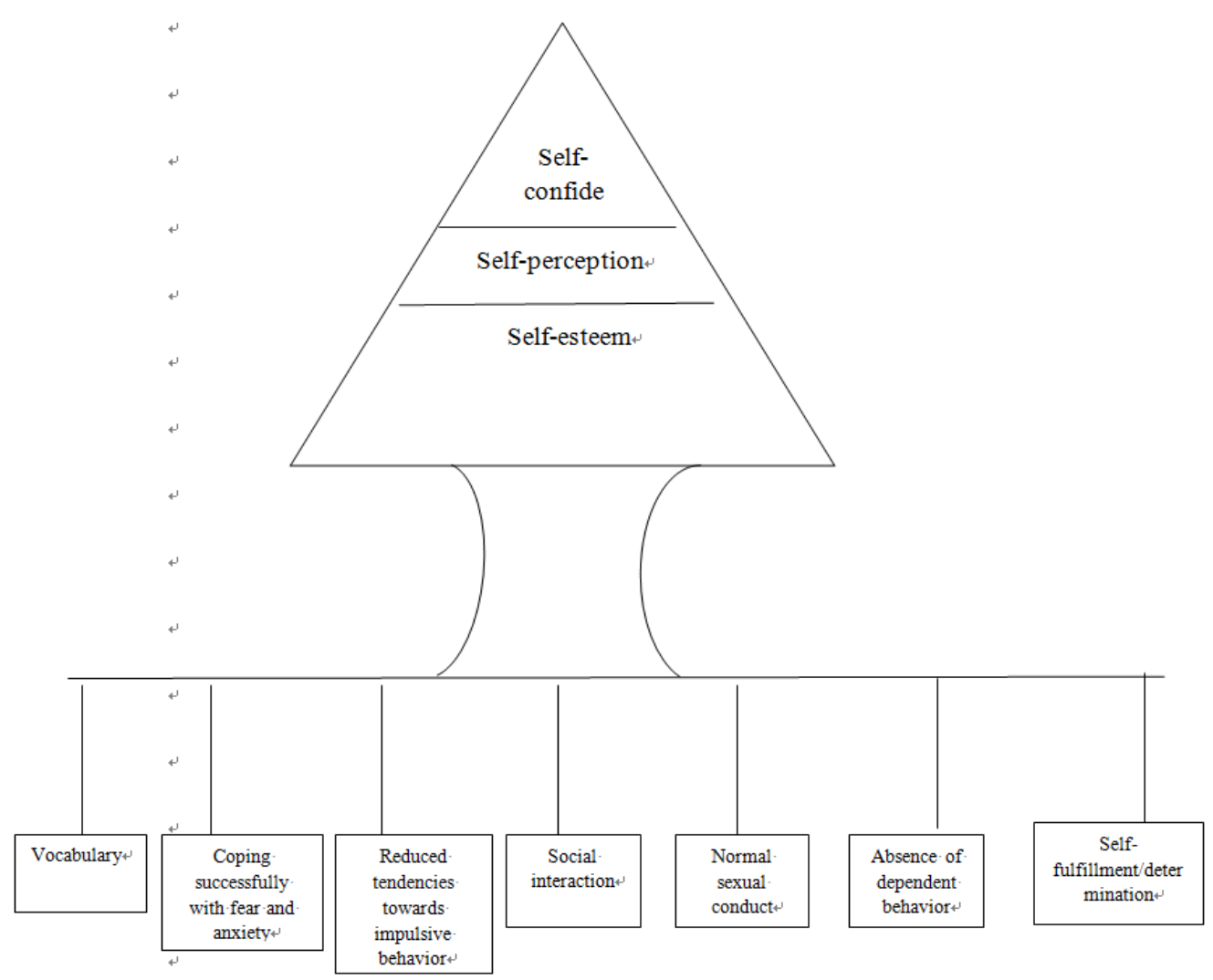

Figure 1. The proposed Self-Fulfillment Scale

This chart is modeled in the form of a tree, with roots that nourish the trunk and the additional components of the tree, while self-fulfillment is naturally located at the top of the tree. Before beginning to constructively describe the journey towards achieving self-fulfillment, I would like to note that during therapy, I often encounter a decline in psychological and personality function among adolescents and adults, which can generally be attributed to a problem that developed along the way. This is related to the concept of distorted thinking described by David D. Burns in his bestseller Feeling Good (1999), which involves dipolar thought processes resulting from impaired development. Burns' theory describes an additional nine types of distorted thinking, which are related to the seven elements presented in the roots of the model above.

This model is an excellent representation for normative development. It does not apply to emotional, psychological, or cognitive difficulties.

The proposed scale consists of three primary elements. The first includes a rich vocabulary and the ability to function well while maintaining relatively low degrees of fear and anxiety. Individuals will tend not to act impulsively and will maintain active social connections. Their sexual conduct is normal, their sexual identities are well-defined, they are not overly dependent on their parents, and they tend to plan ahead and be well organized. The second is the concept of self-awareness, which mediates between these basic elements and the upper element of the model.

When the first stage is sufficiently developed and coherent, it becomes fertile ground for self-awareness. When these two elements are well defined and compatible, the next four elements of the treetop can be developed, beginning with a broad, firm foundation of self-esteem, which nourishes the individual's self-perception. The two combined elements empower the individual so that self-confidence is developed. These forces work together to reach the top of the tree and to enable an enhanced sense of self-fulfillment.

\section{The whole is greater than the sum of its parts}

When a horizontal and vertical connection is formed between the elements, the contribution of each element increases. This is in fact the logical basis for this model. Each element gradually nourishes one or more of the other elements, until it is not only the various elements that are impacted but the entire perception of the situation. When a child suffers from a lack of social interaction, enhancing elements such as verbal skills and linguistic abilities will in turn enhance social skills as well. Thus, an interactive symbiosis is formed that drives and motivates the necessary messages so that energy is focused on supportive and positive development. I refer to this process as a "leveraging coherent connection." 
In family environments that neglect any of these elements, the growing child will intuitively develop a parallel element for purposes of empowerment. This occurs during the early stages, as the roots of the tree as they appear in the diagram are developed to nourish the entire tree. At this stage, survival skills are acquired to trigger the interactive processes that lead to self-awareness. Each family provides its children with the environment necessary to thrive, embraces them, and supplies foundations for developing a firm psychological, emotional, and personal basis for growth.

The processes generally occur naturally between two and seven years of age. Their purpose is to develop thinking models, such as analytic and productive thinking. Once a narrative is developed that provides psychological and emotional support for positive growth, distorted thinking and its negative impacts will be significantly less apparent in the later stages of development.

My model emphasizes the role of self-awareness, as it connects the seven elements that nourish the tree and triggers the process with the treetop, leading to the ultimate goal of self-fulfillment and self-determination.

The third element begins with established self-esteem, which can have a positive impact and provide a strong, confident foundation for further growth. As a result, energy and positive thinking emerge, which interact to produce self-fulfillment and determination.

\section{Components}

1. Vocabulary - This component can be divided into two parts: passive vocabulary, which includes the words that a person can understand and identify in speech and written content, and an active vocabulary, which includes the words that a person is capable of using while speaking or writing. Vocabulary is acquired gradually until the age of one year, and from then on, it is acquired at a faster pace.

2. Coping successfully with fear and anxiety - Anxiety is an emotional impairment that can affect children and adults alike. It is impacted by psychological factors, such as personality traits, life experiences, and lifestyle, as well as by physiological factors, such as genetics and chemical neurological processes. Anxiety refers to both physiological and psychological behaviors. It can evoke physical responses as well as psychological reactions, such as paralysis, disassociation, and even fear of death or insanity. To cope with anxiety, individuals must learn to control their physiological reactions and not treat only the physiological expressions of anxiety (Marom, 2003). In previous research, Lewis and Ramsay (1997) examined longitudinally whether early differences in reactivity to stress are related to self-recognition at 18 months of age, and they found a relation between coping with anxiety or stress and self-recognition. It means that great stress reactivity leads to great attention to the self and earlier self-awareness.

3. Impulsiveness - People who act impulsively act without any rational consideration of the outcome or long-term effects of their behavior, even though they have the knowledge and the cognitive ability to comprehend and assess their actions. Impulsive children seem to lack judgment and are unable to control urges, wait for their turn, or follow instructions (De young, 2013). In certain cases, impulsive behavior may inhibit an individual's ability to integrate within society.

4. Social interaction - Social skills include the interpersonal abilities that enable a person to function within a social framework. Difficulties in acquiring social skills can interfere with important aspects of a person's life. Social skills are acquired over the years and are influenced by three primary elements: social-cultural elements, the direct impact of the child's surroundings, and emotional and cognitive development (Crowne, 2009).

Social interactions are related to self-awareness and self-esteem. People with low self-esteem feel insecure in their relationships and experience social identity threat. Self-awareness has been found to be related to healthy social relationships (Steele 2010).

5. Normal sexual conduct - Sexual conduct is considered normal when it is controlled by the individual and does not inhibit everyday activities, in the same fashion as cultural and religious conduct (Weeks, Holland, \& Waits, 2003).

6. Absence of dependent behavior - Dependence is a person's need to be extensively cared for by another person and to maintain constant contact with that person. This is accompanied by lack of self-confidence and difficulty in making decisions.

7. Organization and planning - Conscious, cognitive steps are taken to plan for future events or to achieve a goal. Effective planning can help a person avoid mistakes or reduce the likelihood of their occurrence. Planning involves choosing the best available option and organizing the selected steps to be taken. The product of planning is the ability to devise plans that will guide the person's behavior and the manner in which that person interacts with the surrounding environment. According to Goleman (1996), planning and organization are 
fundamental components of emotional intellectual development. In previous research, Goleman (1996) postulated that management and organization skills are important and related to one recognizing himself.

8. Self-awareness - This relates to a person or group's attention to individuality, including emotions, thoughts, needs, and desires. Self-awareness is an inherent part of nearly all of a person's daily activities and routines. Self-awareness has been defined as the ability to perceive one's self objectively while maintaining a sense of subjectivity (Prigatano, 1991).

9. Self-esteem - This is described as a personality trait relating to one's overall sense of self-worth and beliefs about the self: for example, an appraisal of one's behavior and beliefs (Cheery, 2015).

The degree of self-esteem is determined by the interaction between how the person is treated by his surrounding environment and how the person interprets that treatment. Self-esteem may develop and change throughout childhood, as a result of both physiological changes and varying interactions with the child's environment. By encountering different aspects of their surroundings, children learn to identify their own characteristics and personality traits and how to assess and to appraise them. Low self-esteem may develop as a result of a large gap between ideal and realistic self-esteem (Cohen, 2012).

10. Self-perception - According to Fitts (1971), the concept of self-perception is an expression of all of the qualities that a child attributes to his or her self. This includes personal issues such as character traits, opinions, and emotions regarding experiences and objects. From a social and family perspective, it is the sense of acceptance or rejection and centrality or marginality within the family or social structure. From an ethical perspective, it describes the child's sense of being "good" or "bad" in the social or religious context in which the child lives. Self-perception has a powerful impact on how children relate to their own performance and on how they perceive the world around them. Children continue to act and develop based on their own perceptions of themselves, and there is a tendency to attribute greater value to experiences that support the child's own self-perception and to deny or distort experiences that conflict with it (Azaria, 1988).

Self-perception among students is reflected by the ratio between each student's aspirations and actual achievements. It is a tool used to assess and represent emotional experiences and well-being that is related to social acceptance as well as successfully coping with developmental-educational challenges (Saada, 2009).

11. Self-confidence - This refers to belief in the individual's ability to succeed, confidence in a selected path, and in decisions made. High self-esteem produces self-confidence and a sense of stability and value.

12. Self-fulfillment and self-determination - According to Rogers (1959), self-determination is an ingrained biological power that has the most significant effect on a person's behavior and function. He claims that babies are born with a powerful urge to realize their unique potential, and that this urge is the basis for additional physiological and psychological urges and motivators. We are able to naturally identify the experiences that will help achieve the goal of self-fulfillment and to distinguish them from the experiences that will distance us from that objective. Emotional well-being is expressed by the ability to make decisions based on this internal sensation (Bitman et al., 1992).

Therefore, the current study argues that much work is needed in this area before one can rely on the existing theories. The current study examines the factors that can leads to self-fulfillment and the sources from where these factors are derived among Arab sector in Israel

\section{Method}

\subsection{Participants}

The data included in the current study were collected from a randomly selected population of psychological patients and healthy children and adults. The total sample was formed by 561 participants ( 263 men and 298 women) with an average age of $20.2(\mathrm{SD}=10.16)$.

The participants were recruited to participate in the study while they visited the Educational Psychological Service clinic in north Israel. Informed consent was obtained for the study.

\subsection{Instruments}

\subsubsection{Self-fulfillment Questionnaire}

This questionnaire was developed in the current study. It has 28 items, and each variable contains four items. The items measure the stages of the self-determination continuum. It uses a Likert-type scale of 5 points, where $0=$ not true for me, and $4=$ very true for me.

Ten items, consisting of subscales, concern the degree to which the participant copes with challenges in his or her life, 
including fear and anxiety, normal sexual conduct, vocabulary, social interaction, reduced tendencies towards impulsive behavior, the absence of dependent behavior, and organization.

These subscale scores were used as indicators for the latent variable self-awareness. Cronbach's alphas for the three subscales were as follows: normal sexual conduct $=.69$, vocabulary $=.79$, and social interaction $=.80$, reduced tendencies towards impulsive behavior $=.75$, coping successfully with fear and anxiety $=.81$, and .75 , the absence of dependent behavior $=.77$, and organization $=.68$.

\subsubsection{Self-awareness}

The awareness questionnaire used in this study was also used in Kay, Cavallo, and Ezrachi (1995).The questionnaire contains 33 items across six domains (physical,

cognitive, memory, behavioral, emotional, and executive).

\subsubsection{General self-esteem}

This variable was measured with the 10 items from the general subscale of the Multidimensional Self-Esteem Inventory (O’Brien \& Epstein, 1989).

It is a widely used scale and has well-established reliability and validity. In this study, the alpha was 77.

\subsubsection{Self-perception}

The Self-Perception Profile is a self-report estimation scale that measures self-perception (Harter 1982). Developed by Susan Harter, it contains 12 items and has five subscales to assess perceived domain-specific competence in scholastics, social acceptance, athletic competence, physical appearance, and behavioral conduct and one scale to assess overall self-worth (global self-worth).

\subsubsection{Self-confidence}

The questionnaire contains 22 items. Each item is scored from 0 (not at all confident) to 10 (extremely confident) (Harter 1982).

\subsubsection{Self-fulfillment}

The questionnaire contains 20 items. Each item is scored from 0 (not determined) to 10 (determined) (March \& ONeill, 1984).

\section{Sociodemographic Variables}

A series of sociodemographic variables were included in the questionnaire: sex, age, and education.

Table 1. Means and standard deviations of the variables in the sample $(\mathrm{N}=561)$

\begin{tabular}{lll}
\hline Variable & M & SD \\
\hline Vocabulary & 4.8 & 1.88 \\
Coping successfully with fear and anxiety & 3.28 & 1.96 \\
Social interaction & 4.11 & 1.22 \\
Normal sexual conduct & 4.5 & 1.28 \\
Reduced tendencies towards impulsive behavior & 4.22 & 1.87 \\
Absence of dependent behavior & 4.3 & 1.26 \\
Organization & 3.55 & 1.24 \\
\hline
\end{tabular}

\section{Procedure}

After the participants had granted permission to participate in the project, they attended a group meeting where the research was explained and were given the questionnaires. All participants were told that participation was voluntary and that no one would ever see their questionnaires; therefore, if they chose not to participate, they could simply not complete the questionnaires. Because the participants were at a group meeting, they had to remain in the room for the full meeting to maintain their anonymity; therefore, their option for not participating was simply to not complete all the questionnaires. Thus, the $11 \%$ who did not provide complete data were considered as subjects who chose not to participate in the study.

\subsection{Statistical Analysis}

To test the fit of the hypothesized model, we followed the three-step approach recommended by Anderson and Gerbing (1988). First, EFA analysis was performed on a pilot data set. Second, to determine whether the indicators were related satisfactorily to the latent variables, a confirmatory factor analysis (CFA) was used for the measurement of the model. In the CFA, the factor loading of one indicator for each latent variable was fixed to 1.0 to establish the metric of the latent variable. Third, we tested the fit of the structural model. 
Table 2. Pearson correlations between the subscales of the study

\begin{tabular}{|c|c|c|c|c|c|}
\hline & Self-awareness & self-esteem & Self-perception & Self-confidence & Self-fulfillment \\
\hline Self- fulfillment & $.32 *$ & $.22 *$ & $.3^{*}$ & $41 .^{*}$ & $.34 *$ \\
\hline Self-awareness & 1 & .24 & .25 & .21 & .22 \\
\hline self-esteem & $.33 *$ & 1 & $.32 *$ & $.32 *$ & $.35^{*}$ \\
\hline Self-perception & .11 & .12 & 14. & .12 & .1 \\
\hline Self-confidence & .08 & .05 & .091 & 1 & .09 \\
\hline Self-fulfillment & 0.45 & 0.2 & .04 & .08 & 1 \\
\hline
\end{tabular}

\section{Results}

The current study used a two-step procedure to examine the study hypotheses. First, to develop the measurement model, a hierarchical confirmatory factor analysis was used. Once an acceptable fitting model was developed, the measurement model was modified to represent the theoretical model and tested through structural equation modeling.

\subsection{Measurement Model}

The results of EFA revealed that 6 factors explained 50.15\% of the total variance (Self- fulfillment, Self-awareness, self-esteem, Self-perception, Self-confidence, Self-fulfillment).

The measurement model was accepted. The relative chi-square (2.30) indicated a favorable fit to the data. The RMSEA value of .05 and the $90 \%$ confidence interval around the RMSEA point estimate $(.04-.06)$ indicated a close fit. The values for the CFI (.66), GFI (.54), and AGFI (.42) indicated that the fit was significant.

\subsection{Structural Model}

The fit indices indicated an acceptable fit for the structural model. The relative chi-square value (2.32) showed a favorable fit to the sample data. The RMSEA value of .05 and the $90 \%$ confidence interval around the RMSEA point estimate (.04-.06) indicated a close fit. The values for the CFI (.66), GFI (.54), and AGFI (.42) indicated a significant fit.

\subsection{Hypothesis Tests}

The data supported all the hypotheses: The coefficient was in the predicted direction. The paths for hypotheses were positive and statistically significant $(\mathrm{p}<.001)$. Results indicated factor loadings, item intercepts, and factor variances and covariances. The results of the structured means analysis indicated significantly greater latent variable means for vocabulary, coping successfully with fear and anxiety, reduced tendencies towards impulsive behavior, social interaction, normal sexual conduct, the absence of dependent behavior, and organization/planning.

In summary, the results of the hypothesis tests provided substantial evidence to conclude that the data supported nearly all aspects of the structural model.

The measurement coefficients (the effects of the latent variable on its indicators and error coefficients) for the seven latent variables measured by multiple indicators (vocabulary, coping successfully with fear and anxiety, reduced tendencies towards impulsive behavior, social interaction, normal sexual conduct, the absence of dependent behavior, and organization/planning) are reported in Table 3.

There appears to be no individual problems with any of the indicators loading satisfactorily. All the indicators had moderate to large measurement coefficients (i.e., all the standardized measurement coefficients were above 0.30 , a frequently used rule of thumb cutoff point). The structural effects on self-awareness, self-perception, self-confidence, and self-fulfillment are reported in Table 3. 
Table 3. Direct, indirect, and total effects on self-fulfillment: Total

\begin{tabular}{|c|c|c|c|c|}
\hline Measure & self-awareness & self-perception & self-confidence & self-fulfillment \\
\hline Vocabulary & 0.55 & 0.17 & 0.22 & $0.38[*]$ \\
\hline $\begin{array}{l}\text { Coping successfully } \\
\text { with fear and } \\
\text { anxiety }\end{array}$ & 0.09 & 0.107 & & $0.37[*]$ \\
\hline $\begin{array}{l}\text { Reduced tendencies } \\
\text { towards impulsive } \\
\text { behavior }\end{array}$ & 0.24 & 0.2 & 0.14 & $0.352[*]$ \\
\hline Social interaction & 0.5 & 0.23 & 0.1 & $0.344[*]$ \\
\hline $\begin{array}{l}\text { Normal sexual } \\
\text { conduct }\end{array}$ & 0.12 & 0.14 & 0.17 & $0.3[*]$ \\
\hline $\begin{array}{l}\text { The absence of } \\
\text { dependent behavior }\end{array}$ & 0.2 & 0.14 & 0.15 & $0.35[*]$ \\
\hline $\begin{array}{l}\text { Organization/ } \\
\text { planning }\end{array}$ & 0.13 & 0.19 & 0.102 & $0.3[*]$ \\
\hline
\end{tabular}

Self-perception and self-confidence were mediating variables in that they moderated the effects of the self-awareness and self-fulfillment variables. As such, the basic variables had indirect effects on self-fulfillment through self-awareness. The direct, indirect, and total effects on self-fulfillment for all the variables are reported in Table 3. In summary, the model has the strongest possible theoretical justification and fits the data in the original model.

As seen in the results in Table 3, all the variables had statistically significant effects on self-fulfillment. Among those variables that had significant effects, all were in the direction predicted. Overall, the table shows that most effects identified in this study were direct effects, although there were some indirect effects.

\section{Discussion}

Of all of the scales and models described by researchers and scholars to achieve self-fulfillment and determination, the leading one was presented by Maslow (1943) and emphasized psychological needs and other stages. The scale that the current study has presented in this paper may be considered as a replacement for Maslow's scale. This new scale is a practical scale, as it is abstract but accurately reflects reality.

There has been considerable controversy about the generalizability of human needs in cultural terms (e.g., Heine, Lehman, Markus, \& Kitayama, 1999). Self-determination or fulfillment theories have posited that there are innate psychological needs for competence, autonomy, and relatedness, which implies that satisfying these three needs would promote motivation and well-being in all cultures. Accordingly, the present study was intended, in part, to examine the proposed self-determination scale.

The results of the current study indicated that the multiple indicators: vocabulary, coping successfully with fear and anxiety, reduced tendencies towards impulsive behavior, social interaction, normal sexual conduct, the absence of dependent behavior, and organization/planning) provided self-fulfillment. Self-perception and self-confidence were mediating variables in that they moderated the effects of the self-awareness and self-fulfillment variables.

The results of the study have supported the proposed scale statistically, and the analyses revealed that the measurement model fit the data in both variables, providing general support for the self-determination current scale. This indicates that the constructs are meaningful in each variable. The hypothesized scale consists of three primary elements. The first includes a rich vocabulary and the ability to function will while maintaining relatively low degrees of fear and anxiety. In this scenario, individuals will tend not to act impulsively and will maintain active social connections. Their sexual conduct is normal, their sexual identities are well defined, they are not overly dependent on their parents, and they tend to plan ahead and be well organized. The second is the concept of self-awareness, which mediates between these basic elements and the upper element of the model.

When the first stage is sufficiently developed and coherent, it becomes fertile ground for self-awareness. When these two elements are well defined and compatible, the next four elements of the treetop can be developed, beginning with a broad, firm foundation of self-esteem, which nourishes the individual's self-perception. The two combined elements empower the individual so that self-confidence is developed. These forces work together to reach the top of the tree and to enable an enhanced sense of self-fulfillment. The whole is greater than the sum of its parts. When a horizontal and vertical connection is formed between the elements, the contribution of each element increases. This is in fact the logical basis for this model. Each element gradually nourishes one or more of the other elements, until it is not only the various elements that are impacted but the entire perception of the situation. When a child suffers from a lack of social interaction, enhancing elements such as verbal skills and linguistic abilities will in turn enhance social skills as well. Thus, an interactive symbiosis is formed that drives and motivates the necessary messages so that energy is focused on 
supportive and positive development. I refer to this process as a "leveraging coherent connection." This scale joins various writers, for example, Kao and Sek-Hong (1997), who have criticized the theories of motivation that focus on the goals and needs of individuals.

The results of the current study joined to previous research, in this case vocabulary was found as related variable to self-awareness (Goodman ,2001). Similarly, coping successfully with fear and anxiety seems to be related to self-awareness Lewis and Ramsay (1997) a relation was found between coping with anxiety or fear and self-recognition. It means that fear reactivity leads to more attention to the self and earlier self-awareness. In addition, Social interactions were found related to self-awareness and self-esteem. People with low self-esteem feel insecure in their social interactions and experience social identity threat (Steele 2010). management and organization skills have postulated as important to self-awareness (Goleman, 1996). Impulsive behavior was related to self-awareness in previous research (De young, 2013). Absence of dependent behavior is related to lack of self-confidence and difficulty in making decisions (Goleman, 1996). normal sexual conduct was found as related variable to self-recognition (Tolman, 2012).

With respect to self-determination theory, first, the very idea of enacting moral obligations brings one face to face with the concept of needs. Second, several previous writers have confused the concepts of individualism and autonomy. Deci, Ryan, Gagne, Leone, Usunov, and Kornazheva (2016) argued that the previous portrayal of autonomy as being essentially the same as individualism and independence is inaccurate with respect to the self-determination theory's concept of autonomy.

Nonetheless, the current study proposes an alternative way of thinking about motivational issues and innate needs.

In family environments that neglect any of these elements, the growing child will intuitively develop a parallel element for purposes of empowerment. This occurs during the early stages, as the roots of the tree (as they appear in the diagram) are developed to nourish the entire tree. At this stage, survival skills are acquired to trigger interactive processes that lead to self-awareness. Each family provides its children with an environment that is necessary for them to thrive, embraces them, and supplies a foundation for developing a firm psychological, emotional, and personal basis for growth.

These processes generally occur naturally between two and seven years of age. Their purpose is to develop thinking models, such as analytic and productive thinking. Once a narrative is developed that provides psychological and emotional support for positive growth, distorted thinking and its negative impacts will be significantly less apparent in the later stages of development.

My model emphasizes the role of self-awareness, which connects the seven elements that nourish the tree and triggers the process with the treetop and the ultimate goals of self-fulfillment and self-determination.

The third element begins with establishing self-esteem, which can have a positive impact and provide a strong, confident foundation for further growth. As a result, energy and positive thinking emerge, which interact to produce self-fulfillment and determination.

The roadmap presented by this new scale allows professionals in the field, such as psychologists, social workers, psychiatrists, and others, to work with children of all ages. The main objective of this model is to serve as a guideline based on professional information that will help parents direct their children in the right directions, as the parents work on one important aspect of development at a time and expertly guide their children from youth into adulthood. In other words, this scale can help parents improve their children's daily performance and affect them in three primary ways:

a. Supported parenting: New parents in today's world require a professional guidebook that will help them correctly navigate parenting practices. In general, parents lack crucial information on how to navigate communication between parents and children, and this is a positive application of parent-child interaction that is designed to increase parents' self-confidence and creativity.

Today, and particularly since the Internet became part of our lives, parents have instant and easy access to large quantities of information on parenting that explains how to guide their children in the best directions. Unfortunately, we all realize the dangers of this method of obtaining information, as plenty of misinformation is available that has an indirect, negative impact on how parents and children communicate.

b. Making the good and effective aspects of child development more accessible: The structure presented here is simple to understand and to use. The fundamental concepts of the scale and its structure are easily understandable and accessible and represent different aspects of human culture. Just being aware of the existence of these concepts and understanding how they interact with each other can trigger processes that have the ability to form desirable bonds between parents and children. 
c. Increased willingness and more active cooperation from children: This scale facilitates bidirectional communication. Parents that base their relationship with their children on the components presented here will be rewarded with their children's willingness to cooperate. This willingness is the result of a desire to achieve more, but most importantly, it is the outcome of the child's sense of stability, confidence, and faith in their parents.

The results from the current study are expected to contribute to a better understanding of how basic psychological characteristics (particularly those related to vocabulary, coping successfully with fear and anxiety, reduced tendencies towards impulsive behavior, social interaction, normal sexual conduct, the absence of dependent behavior, and organization/planning) influence treatment success and reaching self-determination. Future researchers now have the opportunity to explore the utility of the self-determination theory for promoting health behavior change in this most important context. The current hypothesized scale of self-determination should evolve based on rigorous empirical evidence, and applied intervention research is one of the best ways to evaluate and refine the theory.

\section{Limitations and Future Research}

This study has some limitations. First, while the sample was reasonably diverse with regard to the participants' sex, race, sociodemographic status, and age, the participants were all rural residents. Future research should explore whether the same pattern of results can be replicated in other types of communities (e.g., urban).

Moreover, Cultural factors may influence self-fulfillment, self-fulfillment and is influenced by the ethno cultural groups (Singelis, Bond, Sharkey \& Siu Yiu Lai, 1999). However, the current study did not deal with cultural factors and its influence on self-fulfillment and the current findings, future research should deal with ethno cultural factors.

Future research should explore other relevant potential mediating variables of a psychological nature, such as optimism and security.

\section{References}

Alderfer, C. (1972). Existence, relatedness, \& growth. New York: Free Press.

Anderson, J. C., \& Gerbing D. W. (1 988). Structural equation modeling in practice: A review and recommended two-step approach. Psychological Bulletin, 103, 411-423. https://doi.org/10.1037/0033-2909.103.3.411

Author, E. (2013). Principles and issues related to psychological evaluation of parental competence in the Arab Moslem society in Israel. Journal of Health Science, 2, 380-388.

Author, E. (2013). The attitude of the Shari'a courts to parental alienation syndrome: Understanding the dynamics of the syndrome in Arab Society. Journal of Divorce and Remarriage, 54, 537-549. https://doi.org/10.1080/10502556.2013.828982

Author, E. (2014). Therapeutic coping with ADHD among children from the Arab Israeli sector with an emphasis on the therapist-patient relationship. Medicine and Law, World Association for Medical Law. University of Haifa, 33, 119130.

Author. E. (2014). Adaptation in Shari'a law, its impact on the Muslim minority In Israel and the therapist-patient relationship when psychological intervention is necessary. Journal of Health Law, 33, 2134.

Azaria, S. (1988). Developing capabilities and self-esteem. Hed Hagan Journal, Israel Teachers Union, Issue 3, 241245 [Hebrew].

Bitman, I. et al. (1992). Personality: Theory and research. Tel Aviv: Open University [Hebrew].

Burns. D. (1999). Feeling good: The new mood therapy. New York: Harper Collins Publisher.

Cavallo, M. M., Ezrachi, O., \& Vavagiakis P. (1995). The head injury family interview: A clinical and research tool. Journal of Head Trauma Rehabilitation, 10, 12-31. https://doi.org/10.1097/00001199-199504000-00004

Cherry, K. (2015). How do psychologists define self-esteem? http://psychology.about.com/od/sindex/f/what-is-self-esteem.htm Accessed 12.01.2015

Cohen, L. (2012). Motivating learning and self-esteem among school children. Michlol, 28, 5783 [Hebrew].

Crowne, A. K. (2009). The relationship among social intelligence, emotional intelligence and culture intelligence. Organization Management Journal6, 148-163. https://doi.org/10.1057/omj.2009.20

De Young, C. G. (2013). Impulsivity as a personality trait. In K. D. Vohs, \& R. F. Baumeister (Eds.), Handbook of self-regulation: Research, theory, and applications (2nd ed.). New York: Guilford Press.

Deci, E. L., Ryan, M., Gagne, M., Leone, D., Usunov, J., \& Kornazheva, B. (2016). Need satisfaction, motivation, and 
well-being in the work organizations of a former Eastern bloc country: A cross-cultural study of self-determination, University of Haifa Library.

Fitts, W. H. (1971). The self-concept and performance. Monograph 3, Nashville DeDe Wallance Center.

Goleman, D. (1996). Emotional Intelligence. Tel Aviv: Matar [Hebrew].

Goodman, L. (2001). A tool for learning: Vocabulary self-awareness. In C. Blanchfield (Ed.). Creative vocabulary: Strategies for teaching vocabulary in grades L-12 (p. 46). Fresno, CA San Joaquin valley Writing Project. Used with permission.

Harter, S. (1982). The perceived competence scale for children. Child Development, 53, 87-97. https://doi.org/10.2307/1129640

Harter, S. (1985). Manual for the perception profile for children. Denver: University of Denver.

Heine, S. J., Lehman, D. R., Markus, H. R., \& Kitayama, S. (1999). Is there a universal need for positive self-regard. Psychological Review, 106, 766-794. https://doi.org/10.1037/0033-295X.106.4.766

Ilardi, B. C., Leone, D., Kasser, R., \& Ryan, R. M. (1993). Employee and supervisor ratings of motivation: Main effects and discrepancies associated with job satisfaction and adjustment in a factory setting. Journal of Applied Social Psychology, 23, 1789-1805. https://doi.org/10.1111/j.1559-1816.1993.tb01066.x

Kao, H. S. R., \& Sek-Hong, N. (1997). Work motivation and culture. In D. Munro, J. F. Schumaker, \& S. C. Carr (Eds.), Motivation and culture New York: Routledge, (pp. 119-132).

Lewis, M., \& Ramsay, D. S. (1997). Stress reactivity and self-recognition. Child development, 68, 621-629. https://doi.org/10.2307/1132114

Marom, S. (2013). Anxiety disorders. Psychoactualia Journal, Israel Psychological Association, 04, 34-36 [Hebrew].

Marsh, H. W., \& O'Neill, R. (1984). Self-Description Questionnaire III: The construct validity of multidimensional self-concept ratings by late adolescents. Journal of Educational Measurement, 21, 153-174. https://doi.org/10.1111/j.1745-3984.1984.tb00227.x

Maslow, A. (1971). The farther reaches of human nature. New York: The Viking Press.

Maslow, A. H. (1943). A theory of human motivation. Psychological Review, 50, 370-396. https://doi.org/10.1037/h0054346

Maslow, A., \& Lowery, R. (Ed.). (1998). Toward a psychology of being (3rd ed.). New York: Wiley \& Sons.

Mathes, E. (1981). Maslow's hierarchy of needs as a guide for living. Journal of Humanistic Psychology, 21, 69-72. https://doi.org/10.1177/002216788102100406

O'Brien, E., \& Epstein, S. (1989). The multidimensional self-esteem inventory: Professional manual. Odessa, FL: Psychological Assessment Resources.

Pink, D. (2009). Drive: The surprising truth about what motivates us. New York: Riverhead Books.

Prigatano, G. P. (1991). Disturbances of self-awareness of deficit after traumatic brain injury. In G. P. Prigatano \& D. L. Schacter (Eds.), Awareness of deficit after brain

injury: Clinical and theoretical issues. New York: Oxford University Press, (pp. 111-126).

Rogers, C. (1959). A theory of therapy, personality and interpersonal relationships as developed in the client-centered framework. In (ed.) S. Koch, Psychology: A study of a science. Vol. 3: Formulations of the person and the social context. New York: McGraw Hill.

Ryan, R. M., \& Deci, E. L. (2000). Self-determination theory and the facilitation of intrinsic motivation, social development, and well-being. American Psychologist, 55, 68-78. https://doi.org/10.1037/0003-066X.55.1.68

Ryan, R. M., Sheldon, K. M., Kasser, T., \& Deci, E. L. (1996). All goals are not created equal: An organismic perspective on the nature of goals and their regulation. In P. M. Gollwitzer \& J. A. Bargh (Eds.), The psychology of action: Linking cognition and motivation to behavior. New York: Guilford, (pp. 7-26).

Saada, N. (2009). The connection between self-esteem and orientation towards motivation to learn among adolescent Arab students in Israel. Jaama, 13, 375-406 [Hebrew].

Singelis, T. M., Bond, M. H., Sharkey, W. F., \& Siu Yiu Lai, C.(1999). Unpackaging culture's influence on self-esteem and embarrassability. Journal of Cross-Cultural Psychology, 30, 315-341. https://doi.org/10.1177/0022022199030003003 
Steele, C. M. (2010). Whistling Vivaldi and other clues to how stereotypes affect us. New York: Norton.

Thompson, M., Grace, C., \& Cohen, L. (2001). Best friends, worst enemies: Understanding the social lives of children. New York: Ballantine Books.

Tolman, D. (2012). Female adolescents, sexual empowerment and desire: A missing discourse of gender inequity. Sex Roles, 66(11), 746-757. https://doi.org/10.1007/s11199-012-0122-x

Weeks, J., Holland, J., \& Waites, M. (eds). (2003). Sexualities and society: A reader. Cambridge: Polity Press.

\section{Copyrights}

Copyright for this article is retained by the author(s), with first publication rights granted to the journal.

This is an open-access article distributed under the terms and conditions of the Creative Commons Attribution license which permits unrestricted use, distribution, and reproduction in any medium, provided the original work is properly cited. 